\title{
Clinical and Molecular Implications of MED15 in Head and Neck Squamous Cell Carcinoma
}

\author{
David Adler, ${ }^{* \dagger}$ Anne Offermann, ${ }^{* \dagger}$ Rebecca Halbach, ${ }^{* \dagger}$ Wenzel Vogel, ${ }^{* \dagger}$ Martin Braun, ${ }^{* \dagger}$ Glen Kristiansen, ${ }^{\dagger}$ \\ Friedrich Bootz, Jörg Wenzel, ${ }^{\S}$ Ralf Mikut, ${ }^{\top}$ Claudia Lengerke, Markus Reischl, ${ }^{\top}$ Andreas Schröck, ${ }^{\ddagger}$ and Sven Perner*
}

From the Departments of Prostate Cancer Research, ${ }^{*}$ Otorhinolaryngology, ${ }^{\ddagger}$ and Experimental Dermatology,,$^{\S}$ and the Institute of Pathology, ${ }^{\dagger}$ University Hospital of Bonn, Bonn, Germany; the Karlsruhe Institute of Technology, ${ }^{9}$ Institute for Applied Computer Science, Karlsruhe, Germany; and the Department of Biomedicine," University Hospital Basel, Basel, Switzerland

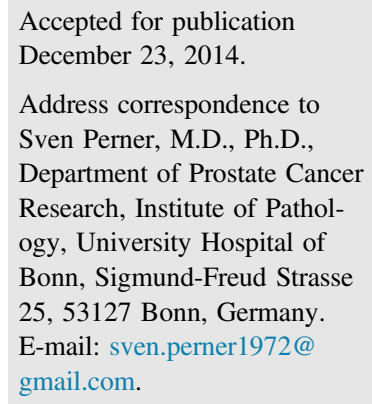

\begin{abstract}
Head and neck squamous cell carcinoma (HNSCC) progression depends on various dysregulated pathways. Regulation of diverse pathways is mediated by the mediator complex. The mediator subunit MED15 is essential for transforming growth factor (TGF)- $\beta$ signaling and involved in breast and prostate cancers. We investigated the implication of MED15 in HNSCC. IHC for MED15 was performed on 324 tissue samples, and TGF- $\beta$ assessed the use of Ki-67 and PSMAD3 as markers. MED15 knockdown followed by proliferation and migration assays, as well as TGF- $\beta 1$ treatment followed by MED15 analysis, was also performed. MED15 was overexpressed in 35\% of primary tumors, $30 \%$ of lymph node metastases, and $70 \%$ of recurrences in contrast to no or low expression in benign tumors. MED15 overexpression in primary tumors from patients who developed recurrences was associated with higher mortality rates and occurred at highest frequency in oral cavity or oropharyngeal tumors. Furthermore, MED15 expression correlated between primary tumors and corresponding lymph node metastases. MED15 correlated with proliferation in tissues, and MED15 knockdown reduced proliferation and migration. We observed an association between MED15 and TGF- $\beta$ activity in tissues because TGF- $\beta$ activation led to increased MED15 expression and reduced PSMAD3 on MED15 knockdown. Taken together, our results implicate MED15 in HNSCC and hint that MED15 overexpression is a clonal event during HNSCC progression. MED15 may serve as a prognostic marker for recurrence and as a therapeutic target. (Am J Pathol 2015, 185: 1114-1122; http://dx.doi.org/10.1016/j.ajpath.2014.12.010)
\end{abstract}

Head and neck cancer includes tumors of the oral cavity, oropharynx, hypopharynx, and larynx, which histopathologically are classified as squamous cell carcinomas (SCCs) in approximately $90 \%$ of all head and neck tumors. ${ }^{1}$ With approximately 600,000 new cases diagnosed each year, head and neck squamous cell carcinomas (HNSCCs) rank as the sixth most common cancer worldwide, ${ }^{2}$ leading to approximately 350,000 deaths per year. ${ }^{3}$ Surgical resection alone or in combination with radiation and chemotherapy is the most common treatment option for HNSCC patients. ${ }^{4}$ However, high rates of recurrence and early metastatic disease ${ }^{1,5}$ lead to a poor prognosis for HNSCC patients. ${ }^{6}$ Furthermore, diagnosis at advanced stages ${ }^{7}$ and lack of available targeted therapeutic approaches result in a survival rate of only $50 \%{ }^{2}$ The main risk factors for HNSCC include lifestyle behavior, such as tobacco use ${ }^{8}$ and alcohol consumption, ${ }^{4}$ and chronic infections with oncogenic human papillomavirus. ${ }^{9}$ Increased knowledge about the underlying genetic alterations ${ }^{10}$ and dysregulated pathways ${ }^{11}$ in HNSCC has led to the identification of promising key targets for therapy in a subset of patients. $^{12}$ The evidence that multiple aberrant pathways account for the progression of $\mathrm{HNSCC}^{11}$ calls for a much deeper understanding of the effect of molecules involved in these signaling pathways on HNSCC progression. Such

\footnotetext{
Supported by German Research Foundation (Deutsche Forschungsgemeinschaft) grants PE 1179/4-1 (S.P.) and LE 2483/4-1 (C.L.), RudolfBecker-Foundation grants H1705701502023319 and T321/20655/2010/kg (S.P.), a medical doctoral fellowship grant (BONFOR) of the Medical Faculty of the University of Bonn (A.O.), and a Gerok Fellowship (BONFOR) of the Medical Faculty of the University of Bonn (M.B.).

D.A. and A.O. contributed equally to this work.

Disclosures: None declared.
} 
knowledge may provide the basis for the development of novel prognostic, predictive, and therapeutic targets for this disease. ${ }^{11}$

Of interest, the regulation of diverse signaling pathways is mediated by a multiprotein complex, called mediator. ${ }^{13}$ Through interactions with regulatory transcription factors on one hand and the Pol II transcription machinery on the other hand, mediator is a key for the regulation of protein-coding genes. ${ }^{13}$ The mediator complex consists of 30 subunits in humans, which are part of the head, the middle or tail modules, or the kinase module. ${ }^{14}$ Altered expression and function of mediator subunits have been implicated in various diseases. ${ }^{15}$ For example, MED15, a subunit within the tail module, ${ }^{14}$ serves as a target for different signaling pathways ${ }^{16-18}$ and plays pathophysiologic roles in several human diseases, ${ }^{19,20}$ including breast cancer. ${ }^{21}$ We recently reported the implication of MED15 in castration-resistant prostate cancer and its direct link to transforming growth factor (TGF) $\beta$ signaling in this disease. ${ }^{22}$ Notably, TGF- $\beta$ signaling is involved in HNSCC, ${ }^{23}$ and a large study reported alterations in SMADdependent TGF- $\beta$ signaling at high frequency and revealed that patients with active SMAD signaling exhibited worse survival rates. ${ }^{24}$ Moreover, abnormalities in SMAD-interacting proteins have been described to contribute to dysregulated TGF- $\beta$ signaling. ${ }^{21,22,25}$ Therefore, we aimed to investigate whether MED15 is implicated in HNSCC and thus further contribute to the understanding of the molecular basis of this disease.

\section{Materials and Methods}

This study was approved by the institutional review board of the University Hospital of Bonn in accordance with the Declaration of Helsinki.

\section{Cohort Design}

The cohort used in this study consists of tissues from 119 patients with HNSCC. Briefly, the cohort contained 113 primary tumor tissues, 85 lymph node metastases, 30 recurrent tumor tissues, and 20 samples of normal squamous epithelium from the head and neck area (benign). For each patient, corresponding tissues were available. Of these, primary tumors with corresponding lymph node metastases were available from 82 patients, and 36 patients developed recurrent tumors. Origins of tissues include oral cavity, oropharynx, hypopharynx, and larynx. Recurrent tumors included locally recurrent tumors in the site of the previous resection and recurrent tumors distant from primary tumor. Clinicopathologic data were available from 108 patients and are provided in Supplemental Table S1. For correlation with clinical data of patients who developed recurrent tumors, we included the 36 patients with relapses from our cohort, as well as additional primary tumors from 72 patients who all developed recurrent tumors. Clinicopathologic data of patients who all developed recurrent tumors for these 108 patients are provided in Supplemental Table S2. Mortality was defined as an outcome end point after a follow-up 1 to 12 years after the first diagnosis. All patients used in this study had their conditions diagnosed and were treated at the University Hospital Bonn in Germany.

\section{IHC}

Immunohistochemistry (IHC) was performed as described previously. ${ }^{22}$ Briefly, staining was performed on sections of paraffin-embedded tissues using a Ventana XT immunostainer (Ventana, Tuscon, AZ). The following primary antibodies were used: anti-MED15 rabbit monoclonal (1:50; clone 11566-1-AP; Proteintech, Chicago, IL), ready-to-use anti-Ki-67 rabbit monoclonal (clone 30-9; Ventana), and anti-pSMAD3 rabbit monoclonal (1:50; clone EP823Y; Abcam, Cambridge, England). Positive and negative controls for MED15 and pSMAD3 antibodies were performed using the same IHC protocol without primary antibodies.

Quantitative analysis of MED15 and Ki-67 expression was performed using Definiens Developer XD software version 2.0 (Tissue Studio, Munich, Germany) as described previously. ${ }^{26}$ Nuclear staining level of MED15 is reflected as an expression score, which is calculated by multiplication of the staining intensity and the index of immunoreactive cells. MED15 expression levels were upfront defined by dividing the expression score into the following groups: no expression (score, $<0.07$ ), low expression (score, $\geq 0.07$ to $<0.2$ ), or overexpression (score, $\geq 0.2$ ) (Supplemental Figure S1). For correlation studies, the full score for MED15 was used. The expression score for Ki-67 expression in tissues reflects the index of proliferating cells. The intensity of positive staining for pSMAD3 was assessed semiquantitatively and scored as either 0 (negative) or 1 (positive). For IHC staining of SCC25 and HSC-3 cell lines, cells were grown on glass slides and fixed with $4 \%$ paraformaldehyde overnight. Then IHC was performed according to the published protocol for cultured cell lines by Cell Signaling Technology (Danvers, MA) using an anti-MED15 rabbit polyclonal antibody (1:50; clone 11566-1-AP; Proteintech) or a ready-to-use anti-Ki-67 rabbit monoclonal antibody (clone 30-9; Ventana).

\section{Immunofluorescence}

HSC-3 and SCC-25 cells were grown on glass slides and fixed with $4 \%$ paraformaldehyde overnight. Immunofluorescence was performed according to the manufacturer's protocol (Cell Signaling Technology) using an anti-MED15 rabbit polyclonal antibody (1:50; clone 11566-1-AP; Proteintech).

\section{Cell Lines and Culture Conditions}

SCC-25 cells were purchased from Leibniz Institute DSMZGerman Collection of Microorganisms and Cell Cultures (Braunschweig, Germany) and HSC-3 cells from JCRB Cell Bank (Japanese Collection of Research Bioresources Cell 
Bank (Osaka, Japan). Cells were maintained in Dulbecco's modified Eagle's medium supplemented with $10 \%$ fetal calf serum (FCS) and 1\% penicillin/streptomycin. Cell lines were authenticated in 2014 using Multiplex Cell Authentication by Multiplexion (Heidelberg, Germany) as described recently. ${ }^{27}$ The SNP profiles matched known profiles or were unique.

\section{siRNA Transfection}

To obtain a transient MED15 knockdown, HSC-3 cells and SCC-25 were transfected with $50 \mathrm{nmol} / \mathrm{L}$ siRNA specific for MED15 using Screenfect A (Genaxxon Bioscience GmbH, Ulm, Germany) according to the manufacturer's instruction. Sequences for the MED15-specific siRNA pool were as follows: 5'-CCAAGACCCGGGACGAAUA-3', 5'-GGGUGUUGUUAGAGCGUCU-3', 5'-GGUCAGUCAAAUCGAGGAU- ${ }^{\prime}$, and $5^{\prime}$-CCGGACAAGCACUCGGUCA- $3^{\prime}$. A scrambled siRNA pool was used as the negative control. Cells were used for further experiments 48 hours after transfection.

\section{Proliferation Assay}

Cell proliferation was assessed using MTT proliferation assay (Roche, Mannheim, Germany). Forty-eight hours after siRNA transfection, HSC-3 and SCC-25 cells were seeded in 96-well plates, and proliferation was measured immediately and after an additional 48, 72, and 96 hours. For each experiment, data are given from three independent experiments performed in technical triplicates.

\section{Western Blot}

Cells were washed with ice-cold phosphate-buffered saline, lyzed in protein extraction buffer, and centrifuged at $16.2 \times g$ for 30 minutes. Protein concentration was measured by BCA protein assay (Pierce, Rockford, IL), subjected to SDS-PAGE analysis, and transferred to polyvinylidene difluoride membranes. Membranes were incubated with primary antibodies specific for MED15 (rabbit monoclonal; 1:200; clone 11566-1-AP; Proteintech), $\beta$-actin (mouse monoclonal; 1:5000; clone A1978; Sigma-Aldrich, St Louis, MO), or pSMAD3 (rabbit monoclonal; 1:1000; clone EP823Y; Abcam) overnight as previously described. $^{22,28,29}$ Then, membranes were incubated with horseradish peroxidase-conjugated secondary antibodies at room temperature for 1 hour. Staining was detected using the ECL Plus chemiluminescence system (GE Healthcare Bio-Sciences, Pittsburgh, PA).

\section{Migration Assay}

Forty-eight hours after siRNA transfection, SCC-25 cells were seeded in 6-well plates and grown until confluence. Cells were treated with $10 \mu \mathrm{g} / \mathrm{mL}$ of mitomycin C (Sigma-Aldrich) for 2
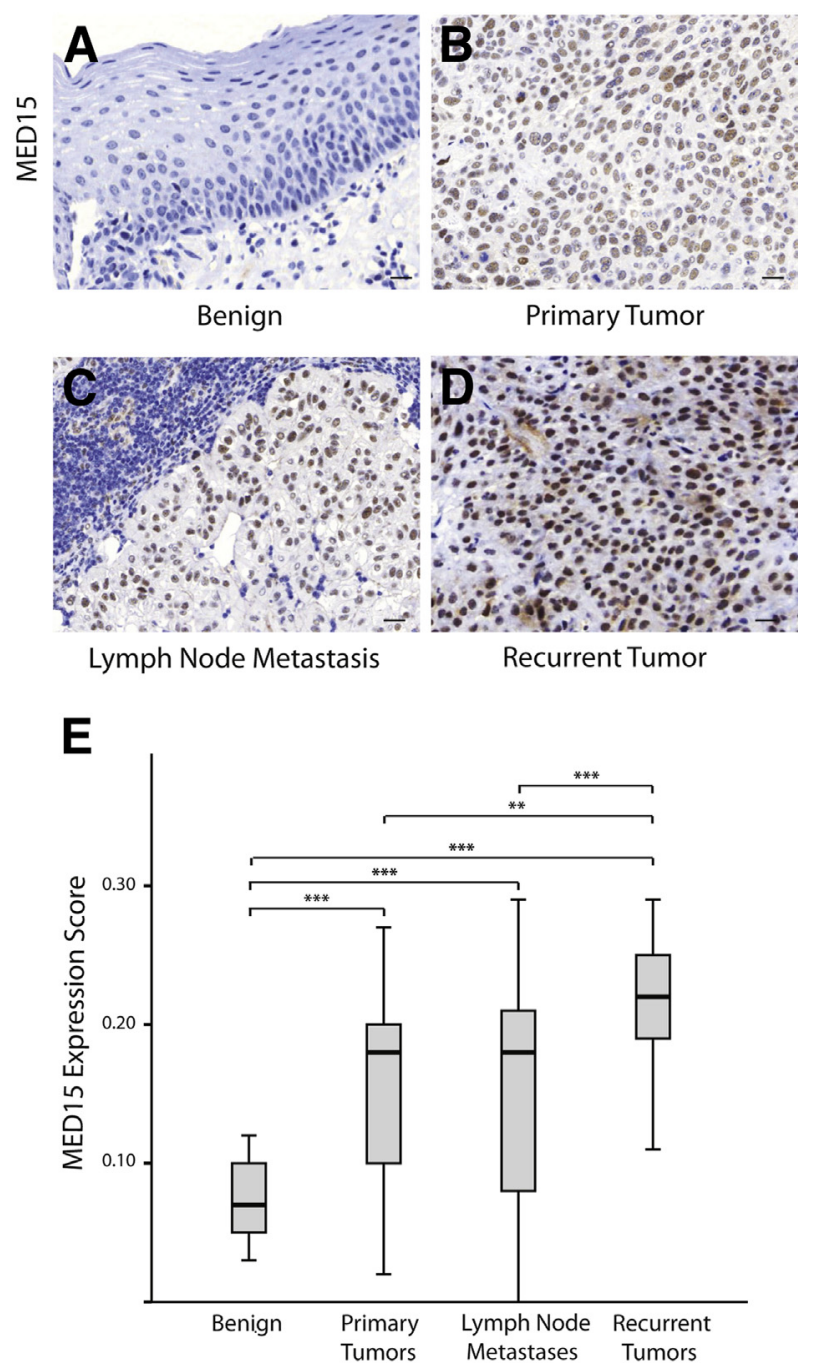

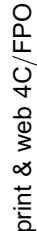

Figure 1 MED15 expression in head and neck tissues. Immunohistochemical staining reveals expression of MED15 in benign epithelium (A), primary tumor (B), lymph node metastasis $(\mathbf{C})$, and recurrent tumor tissues (D). E: Box plots of the median MED15 protein expression reveal increased MED15 expression during cancer progression. ${ }^{* *} P \leq 0.01,{ }^{* *} P \leq 0.001$, independent-sample $t$-test. Scale bar $=20 \mu \mathrm{m}(\mathbf{A}-\mathbf{D})$.

hours to prevent cell proliferation. A wound healing assay was performed in which a scratch was then made across the center of each well using a sterile $200-\mu \mathrm{L}$ pipette tip, and nonadherent cells were washed off with phosphate-buffered saline. Images were taken immediately and after $24,48,72$, and 96 hours.

\section{TGF- $\beta 1$ Treatment}

HSC-3 and SCC-25 cells were seeded in 6-well plates in growth medium to attach overnight followed by serum starvation for 24 hours with medium that contained $2 \%$ FCS. Cells were then treated with 10 or $20 \mathrm{ng} / \mathrm{mL}$ recombinant TGF- $\beta 1$ (Immunotools, Friesoythe, Germany) or with distilled water as control in medium that contained $2 \%$ FCS for 1, 2, or 6 hours. Thereafter, cells were harvested for 
A


B
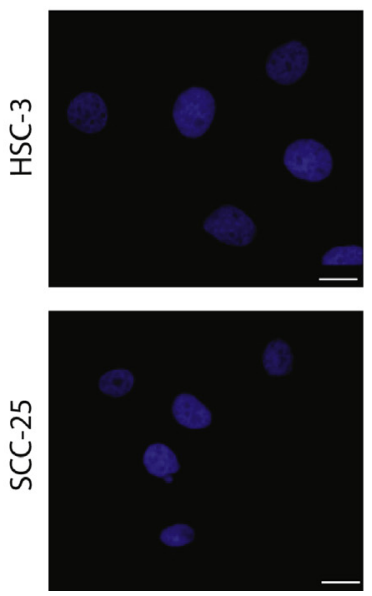

MED15
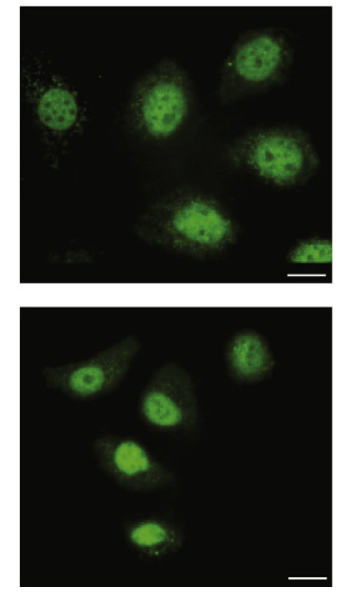

MERGE
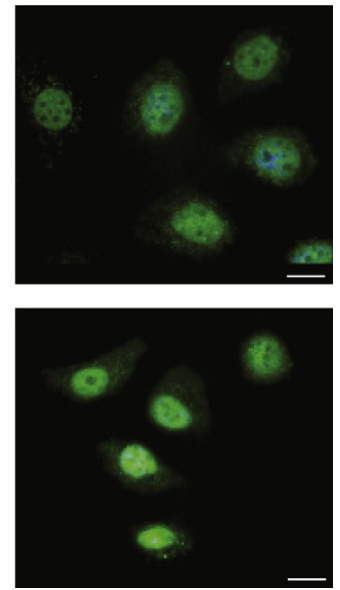

Figure 2 MED15 expression in head and neck squamous cell carcinoma (HNSCC) cells. Immunohistochemical (A) and immunofluorescent (B) staining reveals nuclear MED15 expression in the HNSCC cells HSC-3 and SCC-25. Scale bars: $20 \mu \mathrm{m}$ (A); $10 \mu \mathrm{m}$ (B).

protein analysis. SCC25 cells were transfected with siRNA; after 96 hours, cells were serum starved for 24 hours with medium that contained $2 \%$ FCS after treatment with 10 or $20 \mathrm{ng} / \mathrm{mL}$ of TGF- $\beta 1$ for 1 hour.

\section{Statistical Analysis}

Statistical analysis was performed using SPSS statistical software version 17.0 (SPSS Inc, Chicago, IL).

\section{Results}

\section{MED15 Expression in HNSCC Tissues and Cell Lines}

IHC reveals MED15 to be overexpressed in $35 \%$ of primary tumors, $30 \%$ of lymph node metastases, and $70 \%$ of recurrences, in contrast to no or low expression in all benign tissues (Figure 1, A-D). In particular, we observed that $20 \%$ of primary tumors exhibited no expression of MED15, whereas $45 \%$ harbored low levels of MED15. Furthermore,
$15 \%$ of lymph node metastases exhibited a lack of MED15 expression, and 55\% exhibited low expression levels. In recurrent tumor tissues, MED15 is expressed at low levels in $23 \%$ of samples, whereas a subset of $7 \%$ exhibited no MED15 expression. To illustrate the increase in MED15 expression during HNSCC progression, we compared the median of the MED15 expression score among the different stages (Figure 1E).

To examine the protein expression level of MED15 in HNSCC cells, we performed IHC (Figure 2A) and immunofluorescence (Figure 2B) in the SCC cells, HSC-3 and SCC-25, which are both known to exhibit invasive and metastatic behavior. ${ }^{30}$ Our results indicate that both cell lines harbor a strong nuclear MED15 expression (Figure 2).

\section{Correlation between MED15 and Clinicopathologic Features}

We found MED15 overexpression to be associated with heavy alcohol consumption ( $\chi^{2}$ test, $\left.P \leq 0.01\right)$. Correlation
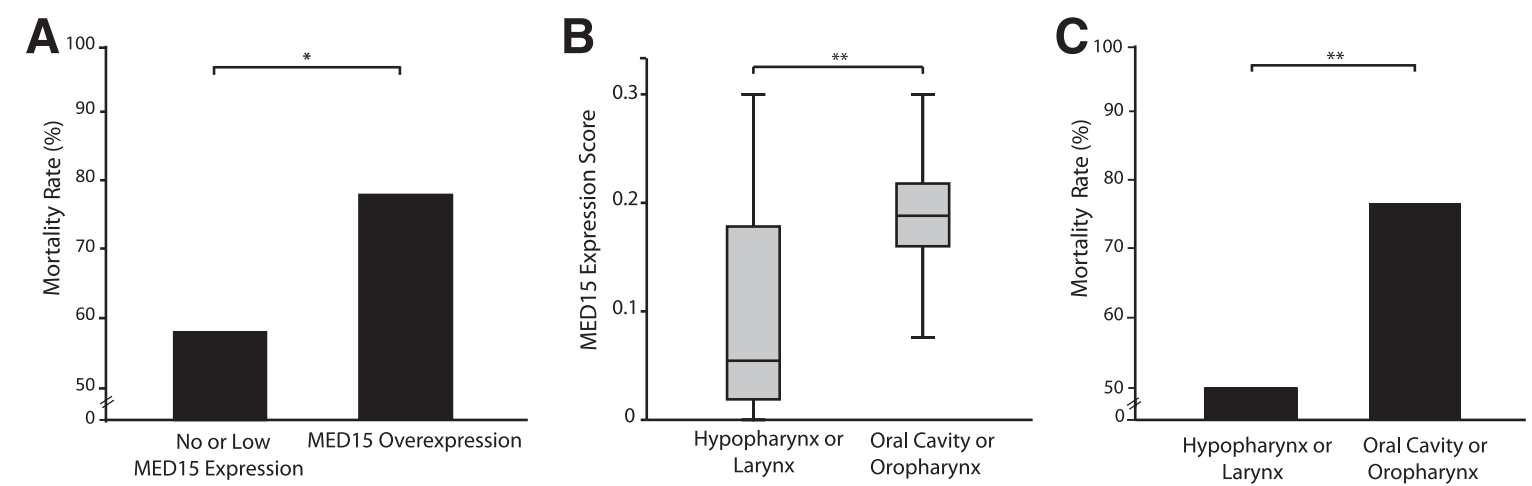

Figure 3 Association of MED15 with mortality rates and site-specific expression. A: Mortality rates of patients harboring MED15 overexpression in primary tumor (78\%) compared with patients with no or low MED15 expression (58\%). B: MED15 expression in tumors localized in the oral cavity or oropharynx compared with the hypopharyngeal or laryngeal tumors. C: Mortality rates of patients with tumors in the oral cavity or oropharynx (77\%) compared with patients with hypopharyngeal or laryngeal tumors $(50 \%)$. ${ }^{*} P \leq 0.05, \chi^{2}$ test $(\mathbf{A}) ;{ }^{*} P \leq 0.01$, independent-sample $t$-test $(\mathbf{B})$ and $\chi^{2}$ test $(\mathbf{C})$. 
analysis with age, sex, tobacco consumption, survival of patients, and pathologic features, such as tumor localization, T-state, presence of lymph node, distant metastases at time of diagnosis, and human papillomavirus status, revealed no significant association. Clinicopathologic characteristics of patients and the corresponding MED15 overexpression status are provided in Supplemental Table S1.

\section{Association of MED15 Overexpression with High Mortality Rate}

To investigate whether MED15 overexpression is associated with clinicopathologic data of a specific subset of patients, we performed IHC on primary tumor tissues from 108 patients who all developed recurrent tumors. As a result, we found a significant association between MED15 overexpression and high mortality rate $(78 \%)$ of patients $\left(\chi^{2}\right.$ test, $\left.P \leq 0.05\right)$ (Figure 3A). On comparing the expression score of MED15 in tumors of different localizations, we found a significantly higher expression of MED15 in tumors that originated from the oral cavity or oropharynx compared with tumors from the hypopharynx or larynx (independent sample $t$-test, $P \leq 0.01$ ) (Figure 3B). Of interest, the mortality rate of patients harboring tumors in the oropharynx or oral cavity was significantly higher than that of patients harboring the tumor in the hypopharynx or larynx ( $\chi^{2}$ test, $P \leq 0.01$ ) (Figure 3C). Furthermore, we found MED15 overexpression to be associated with heavy alcohol consumption ( $\chi^{2}$ test, $P \leq 0.05$ ). Correlation analysis to other clinical data revealed no significance. Clinicopathologic characteristics of patients who developed recurrent disease and the corresponding MED15 overexpression status are provided in Supplemental Table S2.

\section{MED15 Overexpression Is a Clonal Event during HNSCC Progression}

To investigate whether MED15 overexpression may be a clonal event during cancer progression, we compared the expression of MED15 among patients for which we had matched tissues from both primary tumors and their corresponding lymph node metastases. We found that most patients had a concordance for the MED15 overexpression or lack of it between primary tumors and their matched corresponding lymph node metastases (Figure 4A). Furthermore, we observed a significant correlation between the expression scores of MED15 in the lymph node metastases and the corresponding primary tumors (Pearson correlation, $P \leq 0.01$ ) (Figure 4B). Each dot represents the expression score of the primary tumor ( $x$ axis) and the corresponding lymph node ( $y$ axis) for one patient.

\section{Effect of MED15 on Proliferation in HNSCC}

IHC staining for the proliferation marker Ki-67 in HNSCC tissues revealed a significant correlation between MED15 expression and high proliferative activity (Pearson correlation,
A

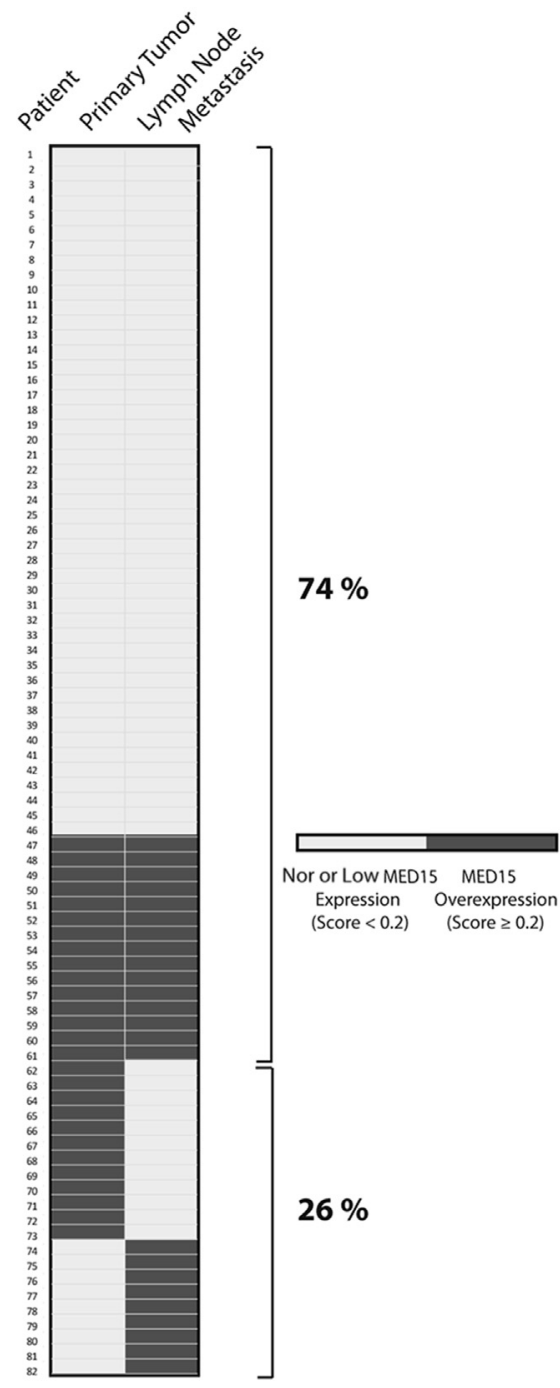

B

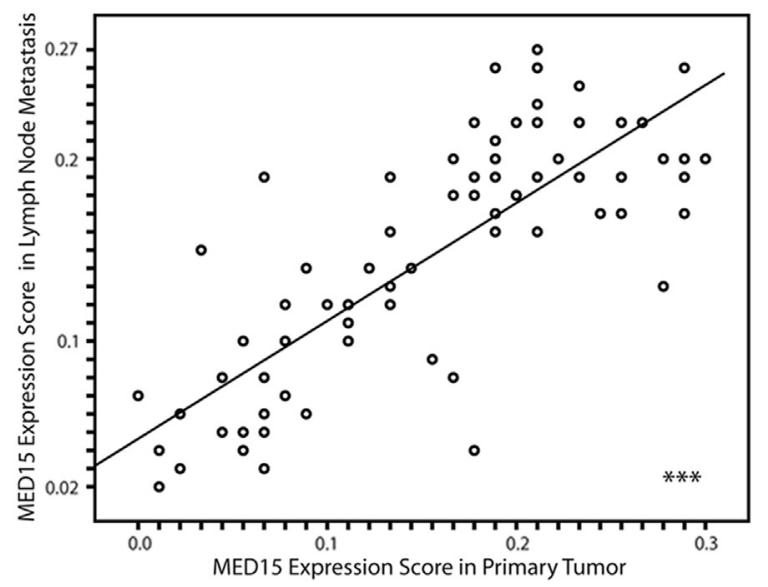

Figure 4 MED15 expression in primary tumors and matched corresponding lymph node metastases. A: Heat map illustrating concordance for MED15 overexpression in primary tumors and their matched corresponding lymph node metastases in $74 \%$ of patients. B: Scatterplot showing a significant correlation between the expression score of MED15 in primary tumors and corresponding lymph node metastases $(r=0.788)$. ${ }^{* * * P} \leq 0.001$, Pearson correlation (B). 

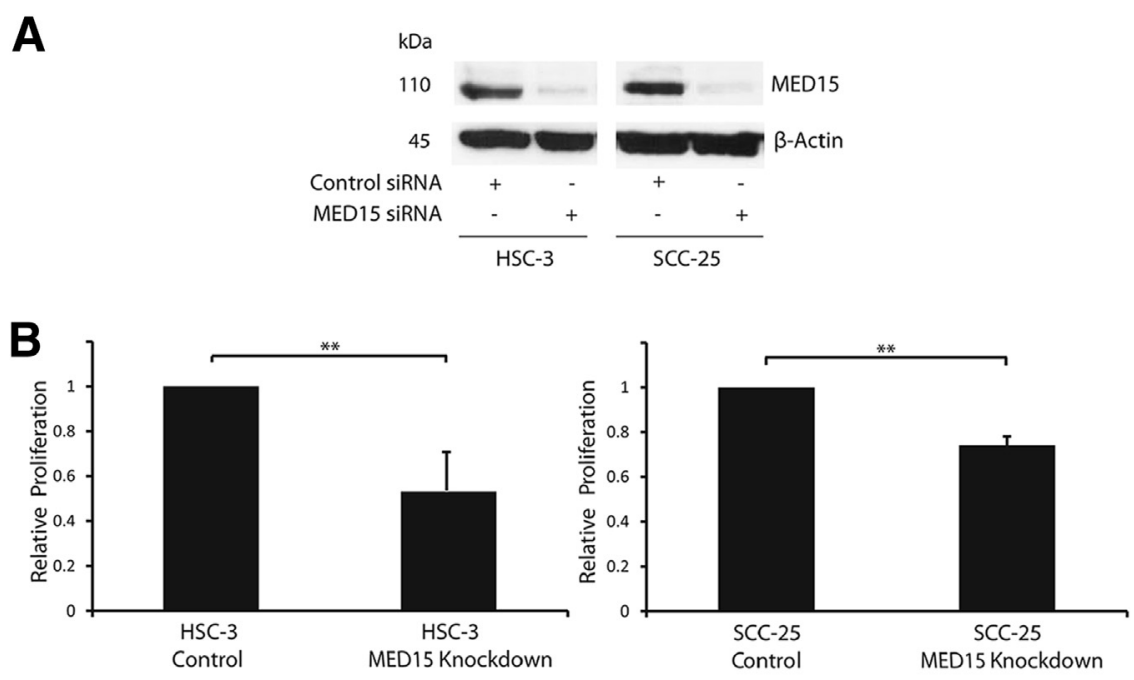

C

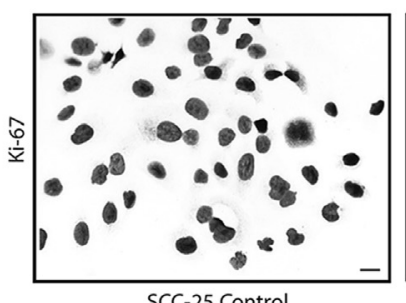

SCC-25 Control



Figure 5 Correlation between MED15 expression and proliferation in head and neck squamous cell carcinoma (HNSCC) tissues and the effect of MED15 knockdown on HNSCC cell proliferation. A: Western blot showing siRNA-mediated MED15 knockdown in HSC-3 and SCC-25 cells. B: MTT proliferation assay of HSC-3 and SCC-25 cells reveals reduced proliferation in MED15 knockdown cells compared with control cells after 72 hours. Absorbance at $595 \mathrm{~nm}$ was normalized to control cells. C: Immunohistochemical staining reveals decreased Ki-67 expression in SCC-25 cells with MED15 knockdown compared with control cells 96 hours after siRNA transfection. ${ }^{* *} P \leq 0.01$, onesample $t$-test (B). Scale bar $=20 \mu \mathrm{m}$ (C).
$P \leq 0.01)$. To investigate whether MED15 affects proliferation, we performed siRNA mediated knockdown of MED15 in the HNSCC cell lines HSC-3 and SCC-25 (Figure 5A). Thereafter, we compared the measured proliferative activity of control and MED15 knockdown cells by MTT proliferation assay and by IHC for Ki-67. We observed a significant reduction in cellular proliferation in MED15 knockdown cells compared with control cells after 72 hours (Figure 5B). In addition, SCC-25 cells with MED15 knockdown exhibited a reduction in the number of Ki-67-positive cells compared with control cells (Figure 5C).

\section{MED15 Affects Migration in HNSCC Cells}

To investigate the effect of MED15 knockdown on migration potential of HNSCC cells, we used the wound healing assay. After 48 hours, SCC-25 control cells colonized the wound at faster rate than MED15 knockdown cells (Figure 6A). In contrast to MED15 knockdown cells, control cells covered the wound completely after 96 hours (Figure 6A).

\section{MED15 Expression Is Linked to TGF- $\beta$ Signaling}

IHC for pSMAD3 as an activation marker for TGF- $\beta$ signaling revealed an association between TGF- $\beta$ signaling activation and MED15 expression (Fisher's exact test, $P \leq 0.05)$. To investigate whether MED15 is implicated in TGF- $\beta$ signaling in the HNSCC cell lines HSC-3 and SCC- 25 , we first analyzed the basal activity status of TGF- $\beta$ signaling and the concomitant expression of MED15 (Figure 6B). Western blot analysis revealed that MED15 and PSMAD3 were co-expressed in HSC-3 and SCC-25 cells (Figure 6B). To examine whether hyperactivated TGF- $\beta$ signaling leads to increased MED15 protein expression, we treated serum-starved HSC-3 and SCC-25 cells with recombinant TGF- $\beta 1$. Phosphorylation of SMAD3 after TGF- $\beta 1$ treatment for 1 hour indicates successful activation of TGF- $\beta$ signaling by the recombinant protein treatment (Figure 6C). In HSC-3 and SCC25 cells, we observed an increased expression of MED15 at protein level in TGF- $\beta 1$ treated cells compared with untreated cells after 6 and 2 hours, respectively (Figure 6D).

The observation that high TGF- $\beta$ activity correlates with strong MED15 expression prompted us to investigate whether MED15 knockdown affects the activity of TGF- $\beta$ signaling. We found that SCC-25 cells with MED15 knockdown expressed lower pSMAD3 levels after TGF- $\beta$ treatment compared with control cells by Western blot analysis (Figure 6E) and IHC (data not shown).

\section{Discussion}

To our knowledge, this study provides the first evidence that a mediator complex subunit is expressed and functionally implicated in HNSCC. We found the mediator subunit MED15 to be overexpressed in HNSCC in contrast to no or low expression in benign epithelium (Figure 1, A-D). 

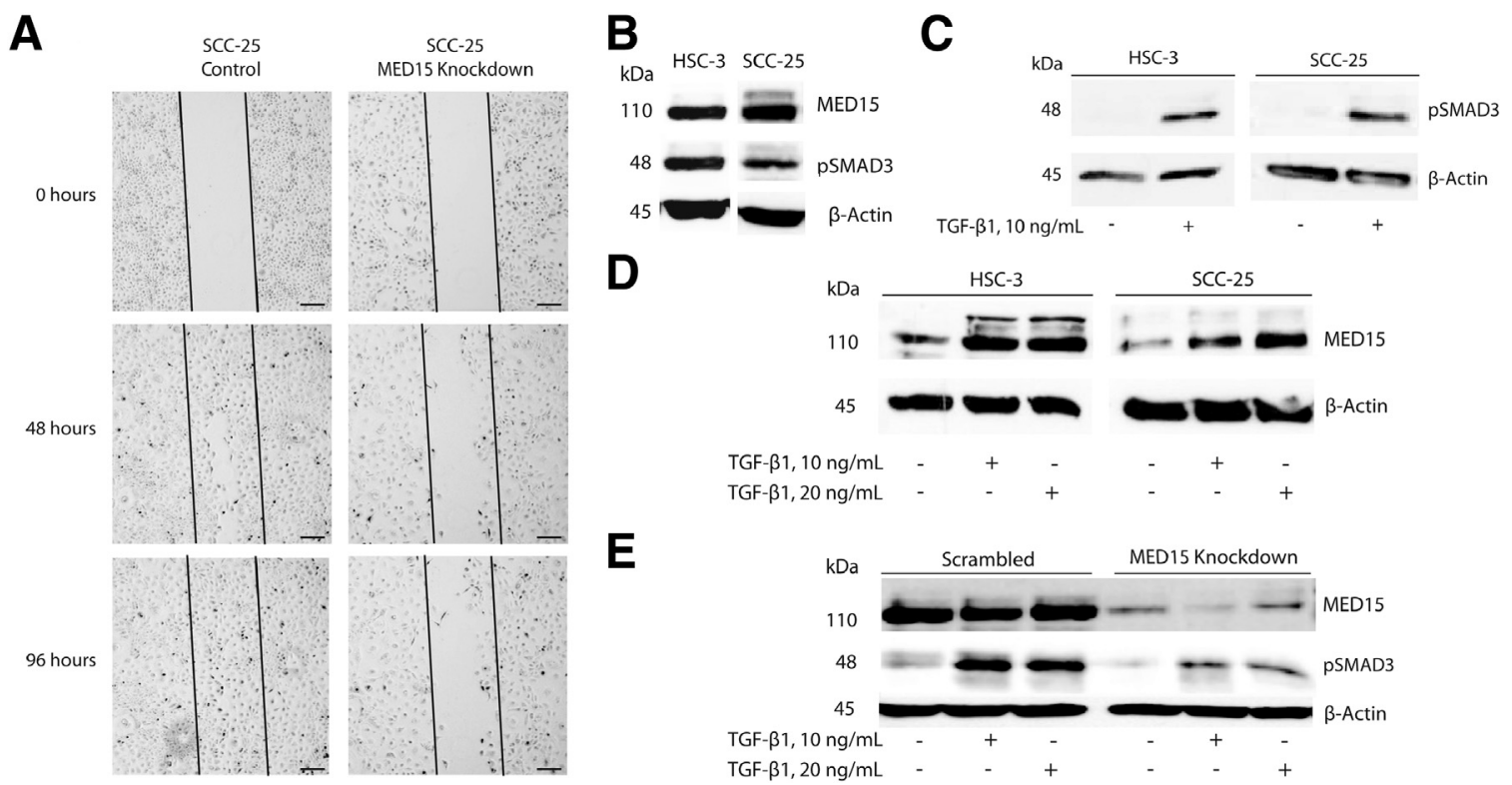

Figure 6 Effect of MED15 on migration and association between MED15 and transforming growth factor (TGF)- $\beta$ signaling. A: Wound healing scratch assay in SCC-25 cells reveals reduced migration of MED15 knockdown cells compared with control cells. Images of cells were taken immediately (0 hour) and at indicated hours after the scratch. B: Western blot of HSC-3 and SCC-25 cell protein lysates exhibiting co-expression of MED15 and pSMAD3. C: Western blot of protein lysates prepared from serum-starved HSC-3 and SCC- 25 cells that were treated with $10 \mathrm{ng} / \mathrm{mL}$ of TGF- $\beta 1$ have increased phosphorylation of SMAD3 after 1 hour. D: Western blot of HSC-3 and SCC-25 cells that were treated with 10 or $20 \mathrm{ng} / \mathrm{mL}$ of TGF- $\beta 1$ reveals increased MED15 expression level after 6 hours in HSC- 3 cells and after 2 hours in SCC- 25 cells. E: Western blot of SCC- 25 cells that were treated with scrambled or MED15 siRNA followed by TGF- $\beta$ treatment for 1 hour reveals reduced pSMAD3 expression in MED15 knockdown cells. Scale bar $=40 \mu \mathrm{m}(\mathbf{A})$.

MED15 is overexpressed in $35 \%$ of primary tumors, $30 \%$ of lymph node metastases, and $70 \%$ of recurrent tumors. In addition, we observed an increased level of MED15 protein expression during progression of the disease (Figure 1E). The observation that MED15 is overexpressed at high frequency in recurrent tumor tissues prompted us to investigate whether MED15 is implicated in the survival of patients who developed relapses. Even though there is a high mortality rate in patients with recurrences (58\%), we found MED15 overexpression to associate significantly with an even higher mortality rate $(78 \%)$ of the patients (Figure $3 \mathrm{~A})$. It is worth noting that survival rates have been reported to differ between HNSCC patients, and oral or oropharyngeal tumors are generally associated with worse outcome than hypopharyngeal or laryngeal tumors. ${ }^{7}$ We consistently observed that tumors in the oropharynx or oral cavity were associated with higher mortality rates compared with tumors in the hypopharynx or larynx (Figure 3C). Of interest, we found a significantly higher expression score of MED15 in tumors that originated in the oral cavity or oropharynx compared with tumors from the hypopharynx or larynx (Figure 3B).

There was a strong correlation between the expression levels of MED15 in primary tumors and their corresponding lymph node metastasis from individual patients (Figure 4B), as well as concordance in $74 \%$ of cases for the presence or absence of MED15 overexpression (Figure 4A). Such observation indicates that MED15 overexpression is likely to be a clonal event in the progression of HNSCC (Figure 4A). Although most patients had concordance, we observed discordance in a subset of patients (Figure 4A). In the case of patients with MED15 overexpression in the primary tumors but without overexpression in the corresponding lymph node metastasis, it is plausible that such observation is because the lymph node metastases that we accessed have developed from another primary tumor clone lacking MED15 overexpression, combined with our inability to have access to all of the lymph node metastases associated with these patients. In the few cases in which the lymph node metastasis exhibits a MED15 overexpression but lacks overexpression in the primary tumor, such observation might be due to the heterogeneity within the primary tumor. Multiple clones within a tumor are known to generate intratumoral heterogeneity in HNSCC ${ }^{31}$ and several primary clones may have led to metastasis. Because we had no access to all the primary clones, we may have matched primary tumors and lymph node metastases from different cell clones, thus observing different levels of MED15 expression.

These findings regarding MED15 overexpression are particularly significant because genetic alterations that provide cells with growth advantages and metastatic potential may be present only in a subpopulation of cells in the primary tumor but increase in tissue from metastases and relapsed HNSCC tumors. ${ }^{32}$ Recurrence and metastatic disease are reasons for poor survival rates of patients with HNSCC and remain a challenge to treat. ${ }^{33}$ Finding molecular markers that may predict the risk for the development of tumor recurrence and presence of metastases might be helpful for early diagnosis and treatment decisions of HNSCC relapse. Results of HNSCC genetic analyses suggest a clonal association between the recurrent primary tumor and the initial primary tumor. ${ }^{34}$ Of interest, overexpression of MED15 is 
detectable in $70 \%$ of recurrent HNSCC tumors (Figure 1E), and our correlation analysis provides evidence that MED15 overexpression is likely to be a clonal event during HNSCC progression (Figure 4A). On the basis of our observations, MED15 overexpression may have the potential to predict the likelihood of the development of recurrences occurring when detected in cancer cells of the primary HNSCC tumors. In support of our tissue findings, we found MED15 to be highly expressed in the HNSCC malignant cell lines HSC-3 and SCC-25 (Figure 2, A and B). Furthermore, we found MED15 expression to correlate with high proliferative activity in HNSCC tissues, and knockdown of MED15 reduced both proliferation (Figure 5, B and C) and migration (Figure 6A) in functional assays. These findings, combined with the high frequency of MED15 overexpression in recurrent HNSCC tumors, indicate that targeting MED15 may serve as a potential therapeutic option especially efficient in patients with advanced disease and tumor recurrence. The development of a small-molecule inhibitor specific for MED15 that blocks the activity of MED15 might be a possible strategy to target intracellular MED15 in patients with HNSCC. Alternatively, a small-molecule inhibitor can be used to disrupt the interaction between MED15 and pSMAD3. Because MED15 is localized in the cytoplasm and nucleus of cancer cells, smallmolecule inhibitors that reach intracellular sites through diffusion present the most effective way of targeting MED15.

Furthermore, our results provide evidence of a direct link between MED15 expression and TGF- $\beta$ signaling in HNSCC (Figure 6, B and D). In HNSCC tissues, we observed a significant association between pSMAD3 and MED15 expression. We found increased MED15 expression in response to hyperactivation of TGF- $\beta$ signaling in HSC- 3 and SCC- 25 cell lines (Figure 6D). In support of these functional findings, similar observations have been reported in breast ${ }^{21}$ and prostate cancer ${ }^{22}$ cells. Findings in breast ${ }^{21}$ and prostate ${ }^{22}$ cancers have revealed that MED15 is required for TGF- $\beta$ transcriptional activation ${ }^{17,21}$ and TGF- $\beta$ regulated downstream effects. ${ }^{17,21,22}$ We found MED15 and pSMAD3 expression to be associated with HNSCC tissue and that cases that lacked MED15 exhibited no pSMAD3 expression. Collectively, these findings indicate that MED15 affects TGF- $\beta$ signaling activation. In many cancer types, including HNSCC, dysregulated TGF- $\beta$ signaling promotes an epithelial-mesenchymal transition-like phenotype through several mechanisms. ${ }^{23}$ This includes regulation of the expression of transcription factors that are involved in epithelial-mesenchymal transition, ${ }^{35}$ and MED15 is known to be essential for TGF- $\beta$ activated gene expression. ${ }^{17,21}$ Several studies revealed that TGF- $\beta$-induced epithelial-mesenchymal transition in cancer cells is SMAD dependent, ${ }^{36,37}$ and expression of activated SMAD in HNSCC tissues correlates with worse survival. ${ }^{24}$ Our observation that pSMAD3 is reduced on MED15 knockdown in SCC25 cells is consistent with similar findings in breast $^{21}$ and prostate ${ }^{22}$ carcinoma cells. Furthermore, TGF- $\beta 1$ stimulation of HNSCC cells increases the migration ability in wound healing assays, ${ }^{35}$ and our results reveal a reduced migration of HNSCC cells and decreased levels of pSMAD3 in response to TGF- $\beta$ stimulation on MED15 knockdown (Figure 6, A and E). Because TGF- $\beta$ signal transduction plays a critical role in HNSCC progression, ${ }^{24,38}$ targeting MED15 based on our results may have a major effect on the progression of HNSCC.

Taken together, our findings implicate the mediator complex through its subunit MED15 for the first time in HNSCC. We further present evidence that MED15 overexpression is a clonal event during HNSCC progression and associates with high mortality in patients with recurrences. Our findings further suggest that MED15 may serve as a prognostic marker for recurrence and as therapeutic target in HNSCC patients with recurrences.

\section{Supplemental Data}

Supplemental material for this article can be found at http://dx.doi.org/10.1016/j.ajpath.2014.12.010.

\section{References}

1. Barnes L, Eveson JW, Reichart P, Sidransky D: Pathology and genetics of head and neck tumours. WHO Classification of Tumours. Geneva, World Health Organization, 2005.

2. Warnakulasuriya S: Global epidemiology of oral and oropharyngeal cancer. Oral Oncol 2009, 45:309-316

3. Kamangar F, Dores GM, Anderson WF: Patterns of cancer incidence, mortality, and prevalence across five continents: defining priorities to reduce cancer disparities in different geographic regions of the world. J Clin Oncol 2006, 24:2137-2150

4. Forastiere A, Koch W, Trotti A, Sidransky D: Head and neck cancer. N Engl J Med 2001, 345:1890-1900

5. Woolgar JA, Triantafyllou A: A histopathological appraisal of surgical margins in oral and oropharyngeal cancer resection specimens. Oral Oncol 2005, 41:1034-1043

6. Boehm A, Wichmann G, Mozet C, Dietz A: Current therapy options in recurrent head and neck cancer. HNO 2010, 58:762-769

7. Jemal A, Siegel R, Ward E, Murray T, Xu J, Thun MJ: Cancer statistics, 2007. CA Cancer J Clin 2007, 57:43-66

8. Hecht SS: Tobacco carcinogens, their biomarkers and tobacco-induced cancer. Nat Rev Cancer 2003, 3:733-744

9. Benson E, Li R, Eisele D, Fakhry C: The clinical impact of HPV tumor status upon head and neck squamous cell carcinomas. Oral Oncol 2014, 50:565-574

10. Stransky N, Egloff AM, Tward AD, Kostic AD, Cibulskis K, Sivachenko A, et al: The mutational landscape of head and neck squamous cell carcinoma. Science 2011, 333:1157-1160

11. Molinolo AA, Amornphimoltham P, Squarize CH, Castilho RM, Patel V, Gutkind JS: Dysregulated molecular networks in head and neck carcinogenesis. Oral Oncol 2009, 45:324-334

12. Du Y, Peyser ND, Grandis JR: Integration of molecular targeted therapy with radiation in head and neck cancer. Pharmacol Ther 2014, 142:88-98

13. Malik S, Roeder RG: The metazoan Mediator co-activator complex as an integrative hub for transcriptional regulation. Nat Rev Genet 2010, 11:761-772

14. Lewis BA, Reinberg D: The mediator coactivator complex: functional and physical roles in transcriptional regulation. J Cell Sci 2003, 116: $3667-3675$

15. Napoli C, Sessa M, Infante T, Casamassimi A: Unraveling framework of the ancestral Mediator complex in human diseases. Biochimie 2012, 94:579-587 
16. Yang F, Vought BW, Satterlee JS, Walker AK, Jim Sun ZY, Watts JL, DeBeaumont R, Saito RM, Hyberts SG, Yang S, Macol C, Iyer L, Tjian R, van den Heuvel S, Hart AC, Wagner G, Naar AM: An ARC/Mediator subunit required for SREBP control of cholesterol and lipid homeostasis. Nature 2006, 442:700-704

17. Kato Y, Habas R, Katsuyama Y, Naar AM, He X: A component of the ARC/Mediator complex required for TGF beta/Nodal signalling. Nature 2002, 418:641-646

18. Thakur JK, Arthanari H, Yang F, Pan SJ, Fan X, Breger J, Frueh DP, Gulshan K, Li DK, Mylonakis E, Struhl K, Moye-Rowley WS, Cormack BP, Wagner G, Naar AM: A nuclear receptor-like pathway regulating multidrug resistance in fungi. Nature 2008, 452:604-609

19. Xiaoli, Yang F: Mediating lipid biosynthesis: implications for cardiovascular disease. Trends Cardiovasc Med 2013, 23:269-273

20. Berti L, Mittler G, Przemeck GK, Stelzer G, Gunzler B, Amati F, Conti E, Dallapiccola B, Hrabe de Angelis M, Novelli G, Meisterernst M: Isolation and characterization of a novel gene from the DiGeorge chromosomal region that encodes for a mediator subunit. Genomics 2001, 74:320-332

21. Zhao M, Yang X, Fu Y, Wang H, Ning Y, Yan J, Chen YG, Wang G: Mediator MED15 modulates transforming growth factor beta (TGFbeta)/Smad signaling and breast cancer cell metastasis. J Mol Cell Biol 2013, 5:57-60

22. Adler D, Menon R, Braun M, Offermann A, Queisser A, Boehm D, Vogel W, Ruenauver K, Ruiz C, Zellweger T, Svensson M, Andren O, Kristiansen G, Wernert N, Bubendorf L, Kirfel J, Biskup S, Perner S: MED15, encoding a subunit of the mediator complex, is overexpressed at high frequency in castration-resistant prostate cancer. Int J Cancer 2014, 135:19-26

23. White RA, Malkoski SP, Wang XJ: TGFbeta signaling in head and neck squamous cell carcinoma. Oncogene 2010, 29:5437-5446

24. Xie W, Aisner S, Baredes S, Sreepada G, Shah R, Reiss M: Alterations of Smad expression and activation in defining 2 subtypes of human head and neck squamous cell carcinoma. Head Neck 2013, 35:76-85

25. Iyer NG, Ozdag H, Caldas C: p300/CBP and cancer. Oncogene 2004, 23:4225-4231

26. Braun M, Kirsten R, Rupp NJ, Moch H, Fend F, Wernert N, Kristiansen G, Perner S: Quantification of protein expression in cells and cellular subcompartments on immunohistochemical sections using a computer supported image analysis system. Histol Histopathol 2013, 28:605-610

27. Castro F, Dirks WG, Fahnrich S, Hotz-Wagenblatt A, Pawlita M, Schmitt M: High-throughput SNP-based authentication of human cell lines. Int J Cancer 2013, 132:308-314

28. Litzenburger BC, Kim HJ, Kuiatse I, Carboni JM, Attar RM, Gottardis MM, Fairchild CR, Lee AV: BMS-536924 reverses IGF-IR- induced transformation of mammary epithelial cells and causes growth inhibition and polarization of MCF7 cells. Clin Cancer Res 2009, 15: 226-237

29. Ding Z, Wu CJ, Chu GC, Xiao Y, Ho D, Zhang J, Perry SR, Labrot ES, Wu X, Lis R, Hoshida Y, Hiller D, Hu B, Jiang S, Zheng H, Stegh AH, Scott KL, Signoretti S, Bardeesy N, Wang YA, Hill DE, Golub TR, Stampfer MJ, Wong WH, Loda M, Mucci L, Chin L, DePinho RA: SMAD4-dependent barrier constrains prostate cancer growth and metastatic progression. Nature 2011, 470: 269-273

30. Erdem NF, Carlson ER, Gerard DA, Ichiki AT: Characterization of 3 oral squamous cell carcinoma cell lines with different invasion and/or metastatic potentials. J Oral Maxillofac Surg 2007, 65: $1725-1733$

31. Mroz EA, Tward AD, Pickering CR, Myers JN, Ferris RL, Rocco JW: High intratumor genetic heterogeneity is related to worse outcome in patients with head and neck squamous cell carcinoma. Cancer 2013, 119:3034-3042

32. Bockmuhl U, Schluns K, Schmidt S, Matthias S, Petersen I: Chromosomal alterations during metastasis formation of head and neck squamous cell carcinoma. Genes Chromosomes Cancer 2002, 33: $29-35$

33. Ho AS, Kraus DH, Ganly I, Lee NY, Shah JP, Morris LG: Decision making in the management of recurrent head and neck cancer. Head Neck 2014, 36:144-151

34. Califano J, Westra WH, Meininger G, Corio R, Koch WM, Sidransky D: Genetic progression and clonal relationship of recurrent premalignant head and neck lesions. Clin Cancer Res 2000, 6:347-352

35. Saito D, Kyakumoto S, Chosa N, Ibi M, Takahashi N, Okubo N, Sawada S, Ishisaki A, Kamo M: Transforming growth factor-betal induces epithelial-mesenchymal transition and integrin alpha3beta1mediated cell migration of HSC-4 human squamous cell carcinoma cells through Slug. J Biochem 2013, 153:303-315

36. Schiller M, Dennler S, Anderegg U, Kokot A, Simon JC, Luger TA, Mauviel A, Bohm M: Increased cAMP levels modulate transforming growth factor-beta/Smad-induced expression of extracellular matrix components and other key fibroblast effector functions. J Biol Chem 2010, 285:409-421

37. Porsch H, Bernert B, Mehic M, Theocharis AD, Heldin $\mathrm{CH}$, Heldin P: Efficient TGFbeta-induced epithelial-mesenchymal transition depends on hyaluronan synthase HAS2. Oncogene 2013, 32 : 4355-4365

38. Prime SS, Davies M, Pring M, Paterson IC: The role of TGF-beta in epithelial malignancy and its relevance to the pathogenesis of oral cancer (part II). Crit Rev Oral Biol Med 2004, 15:337-347 